\title{
Introduction and Follow Up of Ongoing WiRED International Program
}

\author{
Gary Selnow, $\mathrm{PhD}^{1}$; Maryam Othman, $\mathrm{MD}, \mathrm{MPH}^{2}$, William Crano, $\mathrm{PhD}^{3}$ \\ 1 San Francisco State University, San Francisco, CA \\ ${ }^{2}$ Western University of Health Sciences-COMP, Pomona, CA \\ ${ }^{3}$ Claremont Graduate University, Claremont, CA
}

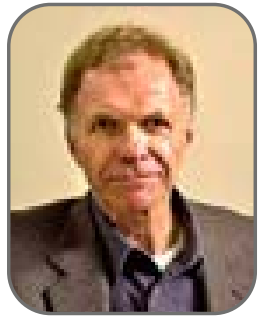

Gary Selnow, PhD

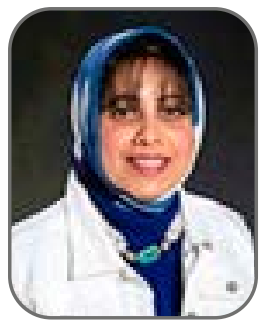

Maryam Othman, MD, MPH

\section{CORRESPONDENCE:}

Gary Selnow, Ph.D.

gselnow@sfsu.edu

DOI: $10.38206 / 130106$
KEYWORDS:

Community Health Worker, Health Education, Prevention and Wellness

\section{ABSTRACT}

As we write this article, the expanding coronavirus pandemic has crushed healthcare systems around the world. Increasingly, ministries of health, non-governmental organizations and hospitals are turning to their reserve workforces - retired professionals, medical and nursing students, and community health workers (CHWs). In underresourced regions, where the options are limited, they are reaching out to these CHWs, a quasi-professional corps of people who play a critical role in the last mile of many health systems. This trained force delivers basic health services, provides community members with health and prevention information, and becomes the eyes and ears for the official healthcare systems. The need for CHWs has surged along with the onset of the coronavirus, but CHWs, like everyone in the healthcare system, are in short supply. The CHW training program that we describe in this paper started out with a different set of motivations and a very different timetable. The coronavirus crisis has imposed changes on our training protocols and greatly accelerated the need for $\mathrm{CHWs}$ in all regions, but especially, underserved regions. In this paper we describe our plans and approaches developed in a very different global environment. At the end, we speculate about how this program will unfold now that training procedures must change, and how $\mathrm{CHWs}$ have become ever more important in community health.

\section{INTRODUCTION}

Physician density varies widely among countries, with around 500 doctors per 100,000 people at the high end and three per 100,000 at the low end. ${ }^{1}$ Although the ratios don't line up neatly with gross domestic product, the lowest physician counts are usually in the poorest regions of Africa, parts of the Middle East and South Asia and segments of Latin America. ${ }^{2}$ With doctors and nurses absent or scarce, common people are left alone to heal the sick, deliver children, address chronic illnesses, all with skills uninformed by the latest medical practices. Moreover, many communities know little about methods of prevention to stave off illnesses.
The number of doctors in low-resource regions is unlikely to grow anytime soon, ${ }^{2}$ and that is where the World Health Organization (WHO) sees CHWs entering the picture, not as substitutes for qualified physicians, but as supplements offering basic clinical services, referrals, health training, and links to the larger healthcare system. CHW services are wide and varied and differ from place to place. A lingering problem has been how to train $\mathrm{CHWs}$ with a standard curriculum while adapting to local differences in health conditions, cultural norms, government requirements and resource availability. 


\section{WHO EXPECTATIONS FOR CHWS}

WiRED International has been working on health education in underserved regions since the late 1990s, and during these past two decades, we have observed the many gaps in health delivery systems that give rise to the need for CHWs. Physicians and medical facilities throughout low-resource communities are in short supply, breathtakingly so in places we have worked in Africa, Latin America, Eurasia, conflict regions of the Middle East, and in earlier years in countries of the former Yugoslavia. We've observed how communities alarmingly are as unprepared for infectious disease outbreaks as they are for routine prevention and management of non-communicable illnesses.

Beyond severe gaps in prevention and medical services, the broken link between official health agencies and dislocated populations may be one of the most perilous and little discussed shortfalls in fragile health systems. Such communities often are isolated economically and geographically from the formal healthcare system, and this separation suppresses the flow of critical information in both directions - to and from the healthcare system and the communities. As a consequence, information about disease prevention and preparedness is slow to diffuse to isolated communities, if the information gets there at all. Moreover, reports about unhealthy conditions within isolated communities often fail to reach outside health agencies. This, of course, has wider population implications beyond the affected communities, when infectious diseases yield dangerous consequences that could have been stemmed with good communication and early interventions. So, broken links between disenfranchised communities and the larger health system have negative consequences for all.

We have been well aware of WHO's convincing arguments in favor of developing a quasi-professional corps of workers who could play a critical role in the health system. This trained force would deliver basic health services, but they would not be physicians. They would provide community members with health and prevention information, but they would not be professional medical educators. They would be the eyes and ears for the official health systems, but they would not be health inspectors. CHWs, thus, provide a range of critical services without the intense focus expected of specialists in medicine, teaching and assessment.

\section{CHW IN-PERSON TRAINING PROGRAM}

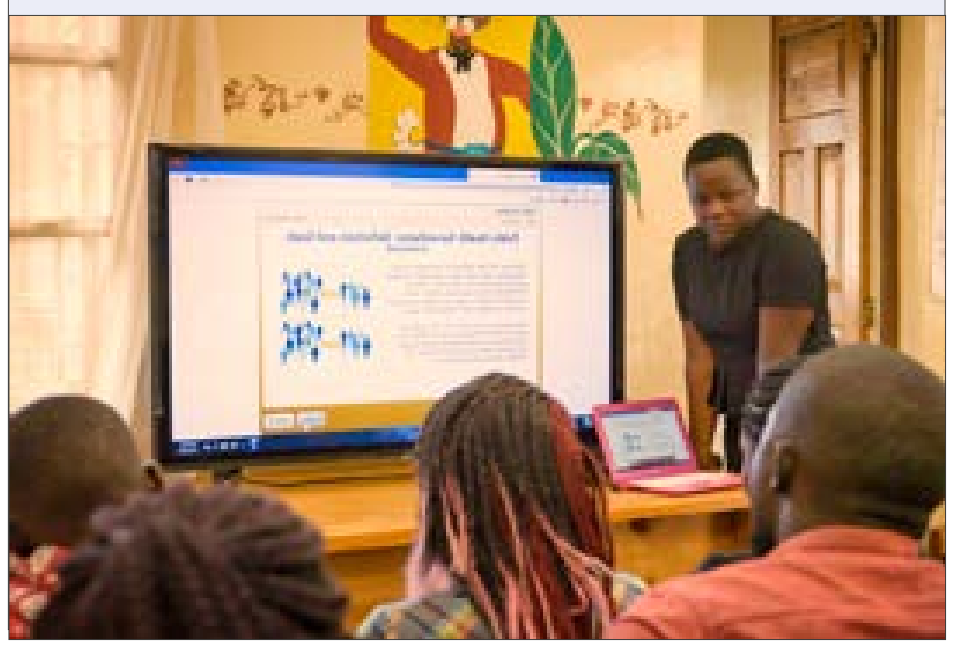

Specifically, WHO has offered these qualifications, functions and expectations for $\mathrm{CHWs}^{3}$

- CHWs can make a valuable contribution to community development and, more specifically, can improve communities with basic health services.

- CHWs teach communities about health and sanitation and stress prevention.

- $\mathrm{CHWs}$ observe populations and local environments, watching for unhealthy and threatening conditions.

- $\mathrm{CHWs}$ can take actions that lead to improved health for everyone and especially for the health of children.

- CHW programs must include continuing health education. Ongoing training ensures that $\mathrm{CHWs}$ remain current in health topics and connected to the health system.

- To be able to make an effective contribution, CHWs must be carefully selected, appropriately trained and - very important — adequately and continually supported.

- CHWs must be firmly embedded in the communities they serve.

- CHW programs succeed in communities that actively want them.

- CHWs must respond to local cultural norms and customs to ensure community acceptance and ownership.

- Examples of successful CHW efforts can be found in programs facilitated by non-governmental organizations or community-based or faith-based organizations. 
- Governments can aid CHW programs by placing them within the overall health sector activities, rather than as a separate activity.

This general WHO list of characteristics and expectations is interpreted and modified by country-level and regional health agencies responsible for $\mathrm{CHW}$ training. We have seen that the training in many locations has become localized and focused on specific conditions and targeted populations, without broad generalizability to other locations. Further, several training programs are U.S.-specific and do not take into account the burdens often faced by underserved and disenfranchised populations. What may work in the rural United States often is not transferrable to rural Kenya or Nicaragua. In this project, we sought to develop a broadbased CHW training program that would be valid across a wide range of low-resource communities where health systems are most fragile and where physicians and nurses are in dangerously short supply.

The low number of physicians per capita in underserved regions is evidence enough of the need to fill the healthcare gap. At present, in many of these places, the burden of healthcare delivery falls to traditional healers or others with scant knowledge of evidence-based medicine. CHWs offer basic clinical services, yes, but WHO conceives of them as playing a larger role in community health. As we noted above, CHWs are tasked as teachers and accordingly play a critical role in prevention. They are the eyes and ears of medical agencies, bringing information to communities, and

\section{CHW IN-PERSON TRAINING PROGRAM}

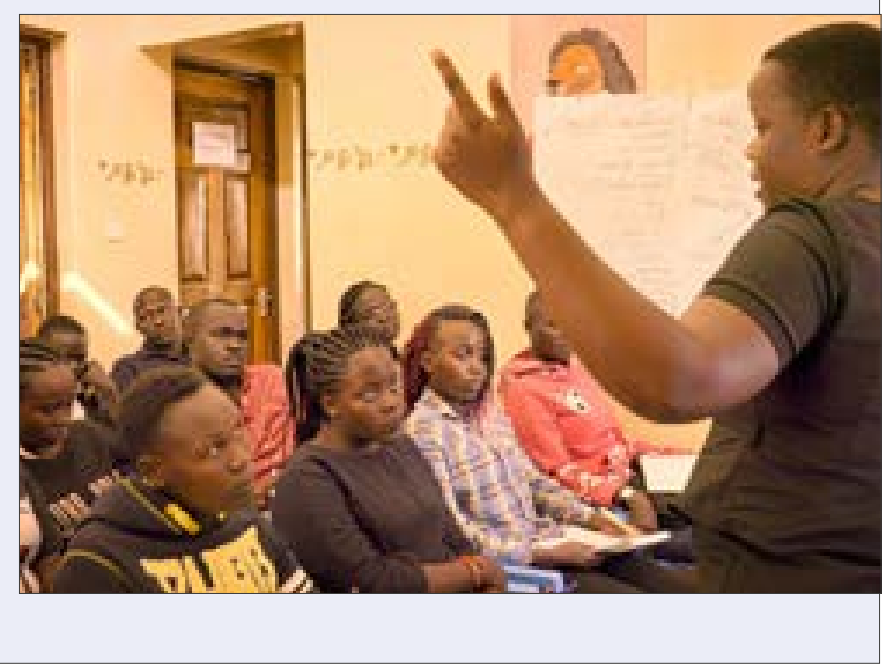

through their surveillance functions reporting community health conditions back to the professional healthcare system. A broad-based CHW curriculum must include all these functions.

We want to stress the role of $\mathrm{CHWs}$ as bridges, as connectors between communities and outside health agencies. By their presence and their interactions, they convey a message that isolated communities are part of the larger population and that their health problems are visible to agencies outside. Training programs have to recognize that CHWs make a symbolic and practical contribution to establishing a bond between the official health system and outlying communities.

\section{CURRICULUM DEVELOPMENT}

Over two years, WiRED has developed a CHW training program that meets $\mathrm{WHO}$ expectations and offers a basic curriculum that is adaptable to any region. Further, it provides a continuing health worker education platform that reinforces the CHW's knowledge base and provides an opportunity to expand skills into new areas of health and community involvement. In this section, we will describe how we developed the program and look at its components.

\section{The process:}

We started by compiling a list of topics based on how WHO sees the functions of $\mathrm{CHWs}$, then submitting the draft curriculum to 12 medical professionals (physicians, nursing professors, and health program administrators) in the United States and abroad for comments and suggestions. Was the curriculum list of topics adequate? Did it miss topics important for $\mathrm{CHW}$ training? Did the list include unneeded items that would be of little practical use? Where should we focus our course material and how should we apportion the training time in a three-week session? Feedback from the panel guided us in retooling the list with a good sense of where to focus the curriculum. We submitted a second draft and received a final round of comments from the panel. The resulting curriculum was divided into two key blocks:

A core block comprised three training sections:

1. Basic health issues (e.g., anatomy, infectious and noncommunicable diseases, healthy practices), 
2. Clinical issues (e.g., vital signs and basic assessment, first aid, medication administration),

3. Communications (e.g., basic communications, teaching and health surveillance).

This core block contains topics of relevance to $\mathrm{CHWs}$ everywhere. All CHWs must understand anatomy, infectious and noninfectious diseases. They all need to take vital signs, teach health, monitor and report health trends within their assigned communities, and carry out training and surveillance activities. There may be minor differences by location, but on the whole, topics in the core are common everywhere. Where local conditions demand, trainers can adapt material in the core block for local applications.

An elective block allows trainers to select from among more than 400 health topics to localize the curriculum. For instance, malaria, cholera and clean water might be central to much of the work in East African locations. Diabetes, Chikungunya and Dengue would be more relevant to areas in Central America. Trainers can identify the most relevant topics for their CHW trainees. In a test of the $\mathrm{CHW}$ program in Kenya described later, trainers recognized the growing relevance of COVID-19, which at the time, was spreading well beyond China. With strong student support, they chose a coronavirus module and a handwashing module as electives.

Why divide the curriculum into a core block and an electives block? We wanted a program that would reflect universal topics for all CHWs and yet would take into consideration local health needs. All CHWs will need to understand basic human health issues. Not all $\mathrm{CHWs}$ will likely need to know about frostbite, schistosomiasis or Ross River virus disease. Those who need to understand these conditions/diseases can study them if they wish. The program, thus, unifies all CHW training around a core of universal topics, while affording each region the opportunity to bear down on the health subjects of greatest relevance to that region.

Design of the training modules: The contents of the modules are evidence-based and peer-reviewed; the structure draws from best learning theory, in which narratives develop several key concepts, then brief quizzes reinforce the key concepts. The sequence is repeated: key concepts then related O\&A. Each module presents a comprehensive, computer-scored final exam, a complete list of reference material and an additional resources section comprising PDFs from authoritative sources allowing further in-depth study. Aware that many users will study this material off the Internet grid, we provide PDFs in the additional resources section rather than Internet links which would not be useful.

Distribution of the training material: In distant, lowresource environments, the best developed course material is useless if you cannot get it to users. And so our next challenge was to find a cost-effective, efficient delivery approach for our program - to get the modules anyplace quickly, even to communities residing off the grid.

For years, we distributed our material on CD-ROMs, and as heavy and bulky as they may be, they're a lot better than books and journals, once the only option for training programs. But then shipping CDs became an increasing burden as our library and our audiences grew. Public postal systems in underserved regions are notoriously unusable, and private delivery services (e.g., DHL, FedEx) are too costly and also unreliable. Technology evolved, and we were happy to adopt the more efficient flash drives, a remarkable improvement over CDs; we could pack the contents of a hundred CDs into one drive weighing less than a house key. But still we faced the challenge of getting a parcel from here to there.

Over our 20 years of health training in low-resource communities, we have seen technologies become smaller, lighter, more engaging and interactive, able to reach far more people much more quickly. We looked for a next-generation technology — past the flash drive — that would deliver these modules to distant communities. Thankfully, the Internet evolved in reach and capacity as phone companies and other service providers expanded throughout the world. Offering modules online is an excellent way to make our material available, and, in fact, many people access the modules directly from our server. However, more than half of our target audience is off the grid or has limited Internet access. That may change in time, but for now the challenge was how can we reach them?

The ideal solution would be a web-based application allowing users to download the modules directly to their devices - laptops, tablets and smartphones. The download would be quick, and the stored modules would be sharable — transferring the files offline to other devices, obviating the need for all users to have an Internet connection. The 
Internet would be instrumental in distribution — so people could acquire the modules - but not essential for the use of the material. Such an app did not exist, so we turned to a volunteer developer who has worked with WiRED for many years. Within a few weeks, Christopher Spirito came up with a windows and iOS version of the app; several months later he completed an Android version.

What was the outcome? People can now download our modules. Why the excitement? It is difficult to overstate the importance of this electronic link for the CHW training curriculum. Think about a small town or village in Africa, Latin America or Asia. The Internet might be available in a nearby community, but it is slow, not always available, and it is expensive for limited budgets. As these things go, outof-the-way locations are often in greatest need of health information and trained $\mathrm{CHWs}$.

With the app (we call it Health Module Access Program or HealthMAP), people from a village can go to a nearby town with an Internet connection, and in a few minutes download to their phones or laptops the $\mathrm{CHW}$ modules or any other health education material they wish. When they travel back home with the modules, they can work entirely offline. Moreover, they can transfer modules from their device to the devices of friends and colleagues. Those people, in turn, can share the modules with others. A single download, therefore, can ripple throughout a population and only the first person needs to get online and only for a brief time.

\section{NEXT STEPS: FIELD RESEARCH}

\section{Preparations}

By mid-2019, we completed the CHW curriculum. It was reviewed and packaged in an interactive format. We had the delivery system ready to go. The next step was to field test the program with trainers and students under the same conditions that would prevail when we rolled out the curriculum. In preparation, we secured the funding and decided on four field test locations that would provide variance in geography, language, economies and social customs. We selected Kenya, India, Nicaragua and Armenia. Our first test site was Kenya.

To set up the Kenya field test, we arranged for two trainers — a clinician and a nurse — with whom we worked via Skype on

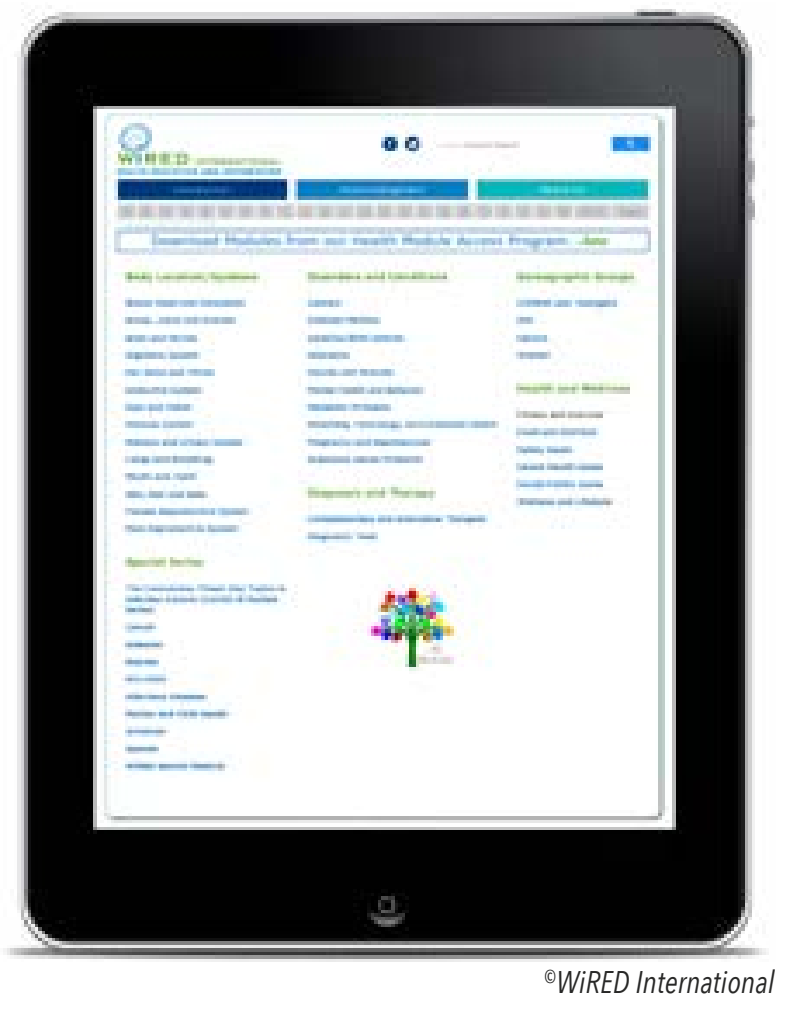

how to teach the curriculum. We selected $15 \mathrm{CHW}$ students, high school graduates in their twenties with no medical or health backgrounds. Finally, we arranged all the logistics necessary for the three-week course.

\section{Training}

The trainers carefully structured the classes around the CHW curriculum. On a large TV monitor we purchased for the project, trainers went through each module in order and offered supplemental material as necessary to answer student questions and stimulate discussion. Unlike most modules in WiRED's library, the modules in this series are not meant to stand alone, but to structure the flow of material and involve trainers to flesh out details, involve students and localize the content where appropriate. The modules, then, provided content, structure and a forum for interaction.

In addition to participating in classroom instruction, students practiced vital sign measurements (e.g., blood pressure, temperature, heart beat). They also studied a module on teaching techniques and then taught a health topic to others in the group; teaching is a critical function required for $\mathrm{CHWs}$. Students during their process evaluation later told us that the hands-on experience gave them confidence in their skills. 
At the end of the course, students took a comprehensive final exam, with items drawn from each module. They also completed a 21-item instrument assessing their attitude toward the course and their views about becoming a $\mathrm{CHW}$ including their self-efficacy.

\section{Outcomes}

A summary review of the attitude measures is included at the end of this paper. In general, it reports that students agreed that they had acquired the knowledge needed to be a CHW, they were confident that their learned skills will help them succeed as a CHW, and they would recommend the course to friends. Responses varied with respect to the difficulty level and pace of the course. All students said they would be proud to talk about their training with potential employers.

Thirteen of 15 students passed the comprehensive knowledge test with $80 \%$ or better. Two missed by a few points. Those two were allowed to study the material for an additional week and retake the exam; both students achieved high passing scores.

\section{Conclusions/recommendations from the Kenya field test:}

- The intensive three-week course is appropriate for this training. We were initially concerned about a fatigue factor, but student interest actually grew through the course.

- Engaging a physician and a nurse as trainers was appropriate. Students often showed interest in peripheral issues beyond the training material, and the medical professionals provided appropriate learning.

- Both trainers and students recommended a more comprehensive review of the body's systems. Accordingly, we have rewritten the anatomy section to include a more in-depth study of anatomy and physiology.

- Students requested a rigorous continuing health worker education program. While this follow-up program had already been developed according to WHO requirements, it was good to know students actively sought it, and we didn't have to "sell" the idea.
- Students were eager to download all the CHW modules to their smartphones, and with these programs, they prepared for classes and studied for the final exam. As we described above, they did not need to get online, they had the material on their phones.

\section{NEXT STEPS . . . NOT AS PLANNED}

After completion of the Kenya research, we had planned in-person tests of the training program in India, Nicaragua and Armenia, in that order. But like every aspect of life, that plan was abruptly derailed by the coronavirus crisis that has swept the world. The authors of this article, who expected to work with the trainers in India and sit in on the sessions as we had in Kenya, were blocked from traveling out of the United States. Moreover, the instructors and students in India could no longer gather in a classroom.

We were disappointed, of course, but we saw an opportunity and developed procedures to run the entire course online observing these steps:

- We will prepare the trainers via instructional technology platforms. Our original plan called for in-person sessions before classes started, but now all preparation will be online.

- Original plans called for monitoring classroom activities by actually sitting in the class. Now we will monitor the class online as well.

- Trainers will conduct the classes live, but they and the students will be connected via a video link. Students will access the class from tablets, which we will provide.

- We will administer the final exam and attitude measures online.

This all-online approach nudges the project further and faster along than we planned originally, but it also enables us to test a program we will use to fill a pressing need for CHWs that is now made even greater by the coronavirus crisis. Many underserved communities are facing this pandemic without the assistance from medical professionals, where $\mathrm{CHWs}$ can have a significant impact on treatment, teaching and surveillance. Moreover, after drugs are developed to address the illness, CHWs will be critical in getting the medications to those who need it and to assisting the vaccination teams who will venture into all communities. 


\section{CONCLUSION}

We set out to create a training program that would be appropriate in most regions of the world. It would offer the fundamental knowledge and skills required by WHO and seen by medical experts as necessary for CHWs. It would go beyond health services to include the wider range of activities including teaching, prevention and health surveillance. We feel strongly about the CHW's role as a bridge between the official health system and communities, and we stress that in our training.

We met the challenge to distribute the program quickly and easily with an application that allows an individual to download the material one time, then share it freely with any number of others, none of whom need to be on the Internet. As fate would have it, that application has now become ever more important as the coronavirus has forced people to keep a distance, preventing in-person training sessions. We wrote earlier that the best content is useless without adequate distribution. That's especially true now; the tools developed by our volunteers have enabled us to reach the far corners of the earth with valuable health training programs.

As we complete this manuscript, we cannot be certain how the coronavirus crisis will play out, and especially in the underserved regions for which our training program was designed. We are fully aware that health systems are in desperate need of trained people to supplement the professional medical corps. Our first field test demonstrated the success of the program to build the knowledge and skills of $\mathrm{CHW}$ s to the point where they would become significant assets in any system reaching out to address growing health needs at this crucial time. The next step, which we could not have envisioned only several months ago, is for us to accelerate the program rapidly to train people who can have a critical impact on a successful outcome of this crisis in communities that otherwise would be underserved, and might go completely overlooked.

\section{AUTHOR DISCLOSURES:}

No relevant financial affiliations or conflicts of interest.

\section{REFERENCES:}

1. World Health Organization's Global Health Workforce Statistics, OECD, supplemented by country data. https://data.worldbank.org/indicator/sh.med.phys.zs

2. World Health Organization. Future public health needs: commonalities and differences between high- and low-resource settings. Background Paper 8. August 2010. https://apps.who.int/medicinedocs/ documents/s17703en/s17703en.pdf

3. World Health Organization. WHO guidelines on health policy and system support to optimize community health worker programs. Geneva, Switzerland: World Health Organization; 2018.

4. https://apps.who.int/iris/bitstream/hand le/10665/275474/9789241550369-eng.pdf 


\title{
EVALUATION OF COMMUNITY HEALTH WORKER TRAINING KENYA, FEBRUARY 2020
}

\author{
By William Crano, Ph.D. \\ Claremont Graduate University
}

Participants were asked to complete a 21-item measure upon completion of the CHW course and the course examination. Questions pertained to participants' attitudes toward the course, their intentions to become a CHW, selective norms, and their perceived behavioral control. Analyses were completed on each of the measures, first to determine the extent to which the items within a construct (e.g., Attitude, Intention) hung together. The coefficient of internal consistency for each of the scales was: .62, .68, .76, and .72 , respectively, which are good given the rather short scales and the small sample. This suggests that the items within each of the scales were tapping a similar construct.

In addition, we ran a t-test on each item, contrasting the obtained score with the midpoint (neutral) scale rating (which was 3). In every case, the obtained item rating, across all 21 items, differed significantly (at $p<.001$ ) from 3 . This means the students' attitudes toward the course were significantly more positive than neutral, that their intentions were positive, and significantly different from neutral, their intentions to become a $\mathrm{CHW}$ were more positive, as were their perceptions of behavioral control (i.e., they felt empowered to become a CHW).

Eighty percent of the participants strongly agreed that after the course, they had the knowledge needed to be a $\mathrm{CHW}$, with the remaining $20 \%$ agreeing. Seventy-five percent strongly agreed that they would succeed in their goal to become a CHW, with the remaining 25\% agreeing. Ninety-three percent were confident that their skills after this training would help them succeed as a $\mathrm{CHW}$, with the remaining $5 \%$ agreeing.

Around $90 \%$ of the participants strongly agreed that the course content was interesting, that the course materials provided valuable information, that the course provides a strong foundation for a career as a CHW, and that they will recommend this course to their friends who are interested in becoming a CHW. The remaining participants agreed. All but two participants either strongly agreed or agreed that the course exceeded their expectations. Responses varied as to the difficulty level of the course and the pace of the course, which is to be expected given the different backgrounds of the participants, with $73 \%$ strongly agreeing or agreeing that the difficulty level was appropriate and $80 \%$ strongly agreeing or agreeing that the pace was appropriate. Two of the 15 responses to the pace question were neutral about the pace.

All participants either strongly agreed (87\%) or agreed that they intend to become a CHW and that the course materials were relevant to their training as a $\mathrm{CHW}$, with $73 \%$ strongly agreeing. Eighty percent strongly agreed that they intend to seek more training in community health, with the remaining agreeing. Two-thirds strongly agreed that becoming a CHW would help them achieve an important personal goal, with the remainder agreeing, except for one who was neutral.

All participants strongly agreed (86\%) or agreed that they will be proud to talk about their $\mathrm{CHW}$ training to potential employers, that their family would be proud if they became a certified CHW (80\% strongly agree) and that becoming a CHW would bring honor to their significant others (e.g., family, friends) (80\% strongly agree). Nearly all students stated that they agree (agree or strongly agree) that their friends would be happy for them to be a CHW and that the community would be happy if they became a CHW. All but one participant strongly agreed that becoming a CHW would be a great benefit to their community, though only $53 \%$ stated that they strongly agreed that their community would welcome them as a CHW, with all but one (who was neutral) stated that they agreed. 